\title{
Puerarin Restore Impairment of Intestinal and Adipose Immunity Response to Influenza Infection in Mice
}

peiping xu ( $\nabla$ xupeiping@gzucm.edu.cn )

Institute of Tropical Medicine, Guangzhou University of Chinese Medicine, Guangzhou, PR China https://orcid.org/0000-0002-6720-7327

Mao-Seng Zeng Institute of Tropical Medicine, Guangzhou University of Chinese Medicine, Guangzhou, PR China

Wen-Di Yu

Institute of Tropical Medicine, Guangzhou University of Chinese Medicine, Guangzhou, PR China

\section{Hui-Xian Wang}

Institute of Tropical Medicine, Guangzhou University of Chinese Medicine, Guangzhou, PR China

Jin-Yuan Liu

Basic Medical College, Guangzhou University of Chinese Medicine, Guangzhou, People's Republic of China

\section{Original Article}

Keywords: Pneumonia, Adipose immunity, Intestinal immunity, Influenza virus, Puerarin

Posted Date: February 4th, 2021

DOl: https://doi.org/10.21203/rs.3.rs-174964/v1

License: (c) (i) This work is licensed under a Creative Commons Attribution 4.0 International License. Read Full License 


\section{Abstract}

Influenza is an acute viral respiratory disease, which can also cause gastroenteritis like symptoms, such as abdominal pain, nausea, vomiting and diarrhea. Immune dysfunction of adipose tissue is involved in the occurrence and prognosis of influenza viral pneumonia. In this study, we analyzed the intestinal and adipose immune responses in mice contracted with influenza virus and found that the impairment of intestinal and adipose immunity response to influenza infection in mice can be restored by puerarin, which is isolated from Gegen (Chinese name).Here, we report that the lung, small intestines(duodenum, ileum, jejunum) and large intestines(colon and rectum) show obvious inflammatory lesions in mice following virus infection, with significantly increased levels of viral load, inflammatory cytokines[interleukin (IL)-6, IL-17, and tumor necrosis factor (TNF)-a],Toll 3, 4 and 9, integrin avb3 and a4, decreased slgA level compared to that of NC group $(P<0.05 \sim 0.001)$. Influenza virus could infect mesenteric lymph nodes and adipose tissue of mice, and the adipokines (e.g., leptin, visfatin, chemokine and adiponectin) of lung and mesenteric adipose tissue are disordered. Puerarin improved the impairment of the intestinal and adipose immune responses in mice infected with influenza virus. Our findings suggest that influenza virus infection can infect adipose tissue and lead to intestinal adipose immune dysfunction in normal-weight mice. The impairment of intestinal and adipose immunity response to influenza infection in mice could be restored by puerarin.

\section{Introduction}

Influenza is an acute respiratory disease caused by influenza virus, with symptoms of fever, headache, stuffy nose and sore throat [1]. Except for these typical symptoms, it can also show abdominal pain, diarrhea, nausea, vomiting and other symptoms similar to gastroenteritis [2,3]. Studies have shown that influenza A virus (e.g. H1N1, H7N2 and H5N1) infection can cause immune damage involving the small and large intestines, which indicates that influenza virus infection can spread to tissues and organs outside the lungs[4]. After influenza virus H1N1 infected mice, there were specific intestinal lesions[2].

Immune function disorder of adipose tissue in obese patients affects their antiviral immunity. During the 2009 H1N1 pandemic, obesity was considered an independent risk factor for the increase in incidence and mortality of influenza[5]. Obese patients were most likely to be infected with influenza A (H1N1) $(71.0 \%)$, and were at high risk of severe cases of influenza[6]. There is accumulation of inflammatory cells and overexpression of cytokines in adipose tissue, which can participate in the inflammatory response to influenza by releasing inflammatory factors[7]. It can be seen that immune dysfunction of adipose tissue is involved in the occurrence and prognosis of influenza viral pneumonia. However, the underlying mechanism of these clinical manifestations in the gut during influenza A virus (IAV) infection is not known. In this study, we analyzed the intestinal and adipose immune responses in mice infected with IAV.

We discovered that influenza virus can infect mesenteric lymph nodes and adipose tissue of mice, and the adipokines (e.g. leptin, visfatin, chemokine and adiponectin) of lung and mesenteric adipose tissue 
are disordered. The impairment of the intestinal and adipose immune responses in mice infected with IAV could be restored by puerarin, which is isolated from pueraria lobata (Gegen,Chinese name) and used to treat fever, cough, cold and other diseases in China [8].

\section{Methods}

\subsection{Viruses and animals}

The strain of influenza virus A/FM/1/47(H1N1) was used, as described previously[9]. BALB/c mice, half male and half female, weighing 13-15g, were purchased from Guangdong Medical Experimental Animal Center ( Guangzhou, China). On the basis of previous studies [9], the dose of IAV induced infection was selected. Except for NC group, mice were intranasally infected with $50 \mu \mathrm{L}$ of $15 \mathrm{MLD}_{50}$ of IAV. In the experiment, $3 \%$ pentobarbital was used to anesthetize mice for euthanasia and the virus was inoculated under ether inhalation anesthesia.

\subsection{Compounds}

Puerarin was purchased from Shanghai Yuanye biotechnology company (batch number: S02M9B54875, Shanghai, China). Puerarin was dissolved in Tween 80 (0.5\% Tween 80$)$ and diluted to the required concentration.

\subsection{In vivo experiments}

The mice were divided into three groups, 10 in each group: normal control group (NC); IAV control group (IAV-C); puerarin group (IAV + puerarin $100 \mathrm{mg} / \mathrm{kg}$ ). The dose of infection of influenza virus was as mentioned above [10]. Puerarin was orally administered to mice 4 hours after infection for 5 days. On the 6th day after infection, the mice were killed and the related samples were collected.

\subsection{Lung index}

The lung index was calculated as described above.

\subsection{Histological analysis}

The lung, duodenum, ileum, jejunum, colon, rectum, mesenteric lymph node (MLN), and mesenteric fat were quickly removed and treated with $10 \%$ PBS buffer, dehydrated with formaldehyde and graded ethanol, and embedded in paraffin. The lungs were cut into $5 \mathrm{~mm}$ pieces and stained with H\&E. As previously described, the histopathological score was blinded to determine the degree of pulmonary inflammation.

\subsection{Viral load analysis}

Quantitative polymerase chain reaction (qPCR) was used to detect the viral load of lung, duodenum, ileum, jejunum, colon, rectum, MLN, and mesenteric fat. As previously described[11], the mRNA expression 
level of H1N1 M gene by qT-PCR are determined. PCR products were expressed according to the normalized expression $\left(2^{-\triangle \Delta \mathrm{ct}}\right)$ [11].

\subsection{Secretive IgA in mucosal washes in IAV-infected mice}

On the $5^{\text {th }}$ day post-infection, mice were killed to collect relevant samples (intestinal and broncho-alveoli washes) and detect secretive $\lg \mathrm{A}(\operatorname{sg} \mathrm{A})$. The total $\lg \mathrm{A}$ in serum, intestinal, nasal and bronchoalveolar lavage fluid were determined by enzyme-linked immunosorbent assay (ELISA) using a mouse IgA ELISIA Kit (Shanghai Enzyme-linked Biotechnology Co., Ltd. Shanghai, China). The ELISA was performed essentially as the manufacturer's directions. Samples were tested at 1:10 ${ }^{2}$ for broncho-alveolar washings, 1:10 ${ }^{6}$ for gut washings. The optical density at $450 \mathrm{~nm}$ was read by an ELISA plate reader (model ELx800, Bio-teck). The standard curve of IgA determination was established with purified mouse IgA.

\subsection{Cytokine in lung and intestinal tissue in IAV-infected mice}

According to the manufacturer's instructions, the concentrations of interleukin (IL) - 6, IL-17, tumor necrosis factor (TNF) - $a$ and interferon (IFN) - $y$ in lung and intestinal tissue homogenate were measured by using mouse ELISA Kit (Shanghai enzyme linked Biotechnology Co., Ltd., Shanghai, China).

\subsection{Adipokines in lung and mesenteric fat tissue in IAV-infected mice}

According to the manufacturer's instructions, the concentrations of leptin, adiponectin, chemokine and visfatin in lung and mesenteric fat homogenate were measured using mouse ELISA Kit (Shanghai enzyme linked Biotechnology Co., Ltd., Shanghai, China).

\subsection{Detection of immune function of lung and intestinal mucosa}

Lung and mesenteric fat were collected, and the levels of Toll receptor (Toll 3,4,9) and integrin (aVB3, a 4) were detected by mouse ELISA Kit (Shanghai enzyme linked Biotechnology Co., Ltd., Shanghai, China) according to the manufacturer's instructions.

\subsection{Statistical analysis}

PASW (windows,ver.18.0,SPSS, Chicago, IL, USA) was used for statistical analysis. The data were expressed as mean \pm SD. One way analysis of variance (ANOVA) and least significant difference test were used to compare the differences among the groups. $\mathrm{P}<0.05$ indicates statistical significance.

\section{Results}

\subsection{Changes of lung inflammation in IAV-infected mice}

Most of the infected mice developed the disease three days after infection, with the symptoms of bristling, curling, tarnished hair, reduced activity, food intake, weight loss, rapid breathing, loose stool and other symptoms. In the NC group, the lung tissue structure was normal and no inflammatory lesions were 
found (Fig.1A,D-E). The lung tissue of IAV-C group showed different degrees of inflammatory lesions, such as inflammatory cell infiltration, bronchiolitis with edema, and perivascular interstitial edema. The lung index in IAV-C group was significantly higher than that in NC group ( $p<0.01)$ (Fig.1B,D-E). Compared to the IAV-C group, puerarin can significantly improve the inflammatory pathological degree of the lung and lessening the lung index $(p<0.01)$ (Fig.1C-E).

\subsection{Pathological changes of intestinal tract in IAV-infected mice}

HE staining showed that inflammatory lesions were found in the small and large intestines of IAVinfected mice, especially in the ileum and colon (Fig. 2-IAV-C). Compared with the normal control group, there were different degrees of inflammatory lesions in the duodenum, jejunum, ileum, colon and rectum in the virus-infected group, especially in the jejunum, ileum and colon (Fig. 2-NC and IAV-C). The local mucosal epithelial cells of the duodenum were necrotic and exfoliated, intestinal villi were destroyed, inflammatory cell infiltration was seen in the mucosal layer, no obvious atrophy was found in the glands in the lamina propria, inflammatory cell infiltration was seen, and some edema was found in the submucosa (Fig. 2-IAV-C-duodenum). The local mucosal epithelial cells of the jejunum were necrotic, and the glands in the lamina propria disappeared and were replaced by new connective tissue. Inflammatory cell infiltration was obvious, and the submucosal structure was loose and edema was present (Fig. 2-IAVC-jejunum). The mucosal epithelial cells of the ileum were necrotic and exfoliated, with local mucosal defects, and the glands in the lamina propria disappeared and were replaced by connective tissue (Fig. 2IAV-C-ileum). Inflammatory cell infiltration was seen in the submucosa. Some of the colonic mucosal epithelial cells were necrotic and exfoliated, with local mucosal epithelial layer defect, lamina propria glands disappeared, the missing mucosal epithelial cells were replaced by new connective tissue, and inflammatory cell infiltration was found in the new tissue(Fig. 2-IAV-C-colon). Local villous epithelial cells of the rectal mucosa were necrotic and exfoliated, mucosal structure was destroyed, inflammatory cell infiltration was visible in the mucosal layer, the gland structure of the lamina propria was damaged, submucosal structure was loose and there was some edema (Fig. 2-IAV-C-rectum). Influenza virus infection can lead to inflammation of mesenteric adipose tissue in mice. The mesenteric lymph nodes were significantly enlarged, and the germinal center was obvious (Fig. 2-IAV-C-mesenteric lymph nodes). In the germinal center, there was proliferation of lymphoblasts and some thickening of the cap-like area. Some atrophy of adipose tissue was observed in mesenteric fat, the number of adipocytes decreased, and a large number of inflammatory cells infiltrated around the fat (Fig. 2-IAV-C-mesenteric adipose tissue). The inflammatory lesions in the puerarin group were lighter than those in IAV-C group, especially in the ileum and colon (Fig. 2-IAV-C-puerarin).

\subsection{Changes of viral load in lung and intestine of IAV-infected mice}

Five days after the mice were infected with influenza virus, viral RNA was found in lung, duodenum, jejunum, ileum, colon, rectum, mesenteric lymph nodes and mesenteric adipose tissue in IAV-C group. The content of viral RNA was significantly higher than that of NC group(Fig.3A-H). Compared with the IAV-C 
group, the level of pulmonary enterovirus titer in puerarin group was significantly lower $(P<0.01)$ (Fig. 3AH).

\subsection{Changes of inflammatory cytokines in lung and intestine of IAV-infected mice}

On the 5th day of influenza virus infection, the levels of inflammatory cytokines TNF-a, IL-6, IL-17 in lung, duodenum, jejunum, ileum and rectum in IAV-C group were significantly increased(Fig.4B-D), and the levels of TNF-a and IL-6 in colon were significantly higher compared to NC group(Fig.4B,D). Puerarin could significantly reduce the above-mentioned tissue cytokine levels(Fig.4B-D). The level of IFN-g was not significantly increased in the lung and intestine tissues, and there was no significant difference between IAV-C group and puerarin group(Fig.4A).

\subsection{Changes of adipokines in lung and intestine of IAV-infected mice}

The level of leptin, visfatin and chemokine in lung, mesenteric lymph node and mesenteric adipose tissue in IAV-C group were significantly higher than those in NC group $(P<0.05-0.001)$ (Fig.5-7). Puerarin could significantly reduce the levels of leptin, lactone and chemokin, and increase the level of adiponectin $(\mathrm{P}<$ 0.01) (Fig. 5-7). The analysis showed that the adiponectin levels in lung, mesenteric lymph node and mesenteric adipose tissue had a significant decrease after 5 days of infection than that in NC group (Fig. 8). Puerarin (100 mg / kg) could inhibit the increase of adiponectin in lung, mesenteric lymph node and mesenteric adipose tissue (Fig. 8A-C, $p<0.05-0.01$ ).

\subsection{Changes of SIgA level in lung and intestine of mice after infection}

Mucosal immune response was evaluated to determine the effects of puerarin. IgA levels in bronchoalveolar lavage and intestinal lavage were detected on the 5th day post-infection. The slgA levels in bronchoalveolar lavage fluid and small intestinal lavage fluid in IAV-C group were significantly lower than those in $\mathrm{NC}$ group $(\mathrm{P}<0.01 \sim 0.001)$ (Fig. 9). The level of slgA in bronchoalveolar lavage fluid and small intestinal lavage fluid of puerarin group was significantly higher than that of IAV-C group (Fig. 9A-B).

\subsection{Changes of mucosal immune function in IAV-infected mice}

On the 5th day after influenza virus infection, the molecular levels of toll receptor (TLR)3, 4, 9 and integrin (aVb3, a 4) in lung, duodenum, jejunum, ileum, colon and rectum in IAV-C group were significantly higher than that in $\mathrm{NC}$ group $(\mathrm{P}<0.05 \sim 0.001$, Fig. 10A-E). Puerarin could significantly improve the molecular levels of toll receptor and integrin in the above tissues $((P<0.05 \sim 0.001$, Fig. $10 \mathrm{~A}-\mathrm{E})$.

\section{Discussion}

Influenza patients often have diarrhea, nausea and other extra-respiratory clinical manifestations[12]. The lungs and large intestine are not only related physiologically, but also affect each other pathologically. Diseases of the lungs and intestines can transmit to each other and even form a vicious circle. In this study, we found that influenza virus not only caused inflammatory lesions in the lungs, but also showed 
inflammatory lesions in the small intestine (duodenum, jejunum and ileum), large intestine (colon and rectum), mesenteric lymph nodes, and mesenteric adipose tissue, especially in the ileum and colon. Influenza-virus-infected mice were accompanied with anorexia, emaciation and loose stools. The results showed that puerarin inhibited the inflammatory changes of IAV-infected mice and improved the immune function of the lungs, intestines and adipose tissue.

Puerarin is a kind of medicinal ingredient isolated from pueraria lobata(Gengen,Chinese name). Pueraria lobata has been used as food source and medicine in China[13]. Puerarin was used to treat fever, cold, cough and flu $[8,14]$. Puerarin is a compound with anti influenza activity by inhibiting NA activity in vivo, in vitro and in vitro[10].

Influenza virus often causes influenza virus pneumonia through respiratory tract mucosal infection. The intestinal and bronchopulmonary lymphoid tissues are important components of mucosal immune system. Secretory $\lg A(\operatorname{sg} A)$ is the main immune molecule of mucosal immune system to resist the invasion of foreign pathogens. Influenza virus infection often leads to immunosuppression and secondary infection of bacteria, often accompanied by gastrointestinal inflammation[4]. This study shows that influenza virus can inhibit the secretion of slgA in bronchoalveolar lavage fluid and intestinal lavage fluid, while puerarin can increase the secretion level of slgA in lung and intestinal mucosa. There is also an immune correlation between the lung and the large intestine. As one of the important mucosal immune activities, mucosal lymphocyte homing has directional selectivity. This selectivity of mucosal lymphocytes is related to the specific recognition of the related receptors on their surface and on the vascular endothelial cells of mucosal sites, such as integrin a4b3, etc. In this study, we found that the levels of integrin avb3 and a4 in the lung and intestine tissues of IAV-infected mice increased, and the contents of TNF-a in bronchoalveolar lavage fluid and intestinal lavage fluid were also increased. Inflammation related injury was induced by the binding of lung gut associated integrin with TNF-a receptor and directional homing migration of immune cells.

TLRs are important in the regulation of inflammatory signal transduction. TNF-a is a proinflammatory cytokine with extensive biological activity that can be produced by a variety of cells[15]. It can induce vascular endothelial cells to express adhesion molecules, promote the infiltration and activation of inflammatory cells, and stimulate the synthesis of inflammatory mediators such as platelet-activating factor, prostaglandin and leukotrienes. In this study, the levels of TLR3, 4 and 9 in the lungs and intestines of IAV-infected mice were significantly increased, together with increased levels of IL-6, IL-17 and TNF-a, which caused or aggravated the pathological inflammation of the lungs and intestines. Puerarin reduced the levels of TLRs and inflammatory factors in lungs and intestines, and alleviated inflammatory damage.

As a part of the intestinal immune system, intestinal fat immunity plays a major role in the pathogenesis of influenza virus pulmonary enteritis[16]. We found that influenza virus infection can cause inflammation of mesenteric adipose tissue in mice. At the same time, the level of adipokines in lung and mesenteric adipose tissue was disordered (leptin, visfatin and chemokine levels were increased, and 
adiponectin level was decreased). Puerarin can regulate adipokines disorder and slow down injury of the lungs and intestines. Adipose tissue, especially intestinal adipose tissue, is generally considered to be an endocrine organ. Adipocytes secrete a variety of hormones and cytokines, such as leptin, adiponectin and resistin, and at the same time, adipose tissue is also an immune organ that plays a major regulatory role in adipose tissue and the immune system[17]. Adipocytes can secrete TNF-a, IL-6 and other inflammatory factors, and their expression and plasma level are related to the amount of body fat [18]. Many adipokines, including leptin, adiponectin, visfatin and resistin, not only mediate innate immune defense by activating a variety of innate immune cells, but also participate in adaptive immunity by mediating the proliferation and activation of immune cells and B lymphocytes [19]. The adipose tissue of obese patients has low-grade inflammation, and the immune function of intestinal fat may be disordered, which makes obese patients most likely to be infected with influenza A virus. Obese patients with influenza A virus infection have more severe symptoms and higher mortality. Adipose immune dysfunction is involved in the occurrence and prognosis of influenza virus pneumonia[7]. Leptin, as a proinflammatory mediator, plays a major role in innate and acquired immune responses [20]. Adiponectin is a cytokine synthesized almost entirely by adipocytes and has anti-inflammatory effect[21]. Visfatin is produced in large quantities by adipocytes and can affect the production of pro-inflammatory (IL-6, TNF-a and IL-1b) and anti-inflammatory cytokines(IL-10)[22]. Chemokines can regulate the production of adipocytes. Through G-protein coupled receptor and chemokine like receptor (CMKLR)-1 signaling pathway, with the participation of chemokine receptors, it acts on immune cells (e.g.monocytes, dendritic cells and natural killer cells) and exerts its chemotactic activity [23].

Mice infected with influenza virus have a low degree of adipose tissue inflammatory damage and immune dysfunction. When the influenza virus invades the body, the inflammatory cells in the infected mice are easily activated, and secrete a large number of inflammatory mediators and adipokines, which further promote the activation of inflammatory cells, and gradually develop into a cascade of inflammatory factors[24]. In this study, the levels of leptin, visfatin and chemokines in the lungs and intestines of IAV-infected mice were significantly increased, together with decreased levels of adiponectin, which caused pathological inflammation of the lungs and intestines. Puerarin can regulate the immune function disorder of adipose tissue.

In conclusion, influenza virus infection can infect adipose tissue and lead to intestinal adipose immune dysfunction in normal-weight mice. Impairment of intestinal and adipose immunity response to influenza infection in mice could be restored by puerarin. However, the specific mechanism of intestinal immune damage caused by respiratory tract influenza virus infection is still unclear, and further research is needed.

\section{Compliance with ethical standards}

None of the authors have any financial interests or conflict of interest with this article. The study was approved by the Animal Care and Use Committee of the Guangzhou University of Chinese Medicine (Guangzhou, China). Humane endpoints are chosen in the experimental animals (e.g., survival study) via 
euthanasia and use of anesthesia. Compliance with regulations for the administration of affairs concerning experimental animals of Guangdong Province (2010,No.41).

\section{Declarations}

\section{Acknowledgement}

We thank International Science Editing (Co. Clare, Ireland) for editing this manuscript. This work was partly supported by a Natural Science Foundation of China grant (81470186).

\section{References}

1. Mauad, T., et al., Lung pathology in fatal novel human influenza A (H1N1) infection. Am J Respir Crit Care Med, 2010. 181(1): p. 72-9.

2. Shu, Y., et al., Avian influenza A(H5N1) viruses can directly infect and replicate in human gut tissues. J Infect Dis, 2010. 201(8): p. 1173-7.

3. Zhang, S., et al., Small intestinal injury in mice infected with respiratory influenza A virus: evidence for virus induced gastroenteritis. Biotechnol Lett, 2015. 37(8): p. 1585-92.

4. Wang, J., et al., Respiratory influenza virus infection induces intestinal immune injury via microbiotamediated Th17 cell-dependent inflammation. J Exp Med, 2014. 211(12): p. 2397-410.

5. Louie, J.K., et al., A novel risk factor for a novel virus: obesity and 2009 pandemic influenza $A$ (H1N1). Clin Infect Dis, 2011. 52(3): p. 301-12.

6. Dawood, F.S., et al., Emergence of a novel swine-origin influenza A (H1N1) virus in humans. N Engl J Med, 2009. 360(25): p. 2605-15.

7. Green, W.D. and M.A. Beck, Obesity Impairs the Adaptive Immune Response to Influenza Virus. Ann Am Thorac Soc, 2017. 14(Supplement_5): p. S406-S409.

8. Wu, M.S., et al., Mechanism of action of the suppression of influenza virus replication by Ko-Ken Tang through inhibition of the phosphatidylinositol 3-kinase/Akt signaling pathway and viral RNP nuclear export. J Ethnopharmacol, 2011. 134(3): p. 614-23.

9. Ye, Y., et al., Polygalasaponin F treats mice with pneumonia induced by influenza virus. Inflammopharmacology, 2020. 28(1): p. 299-310.

10. Wu, F., et al., A new coronavirus associated with human respiratory disease in China. Nature, 2020.

11. Li, Y., et al., Intranasal co-administration of 1,8-cineole with influenza vaccine provide crossprotection against influenza virus infection. Phytomedicine, 2017. 34: p. 127-135.

12. Ison, M.G., Clinical use of approved influenza antivirals: therapy and prophylaxis. Influenza Other Respir Viruses, 2013. 7 Suppl 1: p. 7-13.

13. Prasain, J.K., et al., Profiling and quantification of isoflavonoids in kudzu dietary supplements by high-performance liquid chromatography and electrospray ionization tandem mass spectrometry. $\mathrm{J}$ Agric Food Chem, 2003. 51(15): p. 4213-8. 
14. Zhou, Y.X., H. Zhang, and C. Peng, Puerarin: a review of pharmacological effects. Phytother Res, 2014. 28(7): p. 961-75.

15. Hodge, G., et al., Acute lung transplant rejection is associated with localized increase in T-cell IFNgamma and TNFalpha proinflammatory cytokines in the airways. Transplantation, 2007. 84(11): p. 1452-8.

16. Moschen, A.R., et al., Visfatin, an adipocytokine with proinflammatory and immunomodulating properties. J Immunol, 2007. 178(3): p. 1748-58.

17. Weidinger, C., et al., Adipokines and Their Role in Intestinal Inflammation. Front Immunol, 2018. 9: p. 1974.

18. Moorthy, A.N., et al., Effect of High-Fat Diet on the Formation of Pulmonary Neutrophil Extracellular Traps during Influenza Pneumonia in BALB/c Mice. Front Immunol, 2016. 7: p. 289.

19. Fernandez-Real, J.M. and J.C. Pickup, Innate immunity, insulin resistance and type 2 diabetes. Diabetologia, 2012. 55(2): p. 273-8.

20. Lago, F., et al., Adipokines as emerging mediators of immune response and inflammation. Nat Clin Pract Rheumatol, 2007. 3(12): p. 716-24.

21. Kong, Y., et al., New insights into different adipokines in linking the pathophysiology of obesity and psoriasis. Lipids Health Dis, 2019. 18(1): p. 171.

22. Sawicka, K., et al., Visfatin and chemerin levels correspond with inflammation and might reflect the bridge between metabolism, inflammation and fibrosis in patients with systemic sclerosis. Postepy Dermatol Alergol, 2019. 36(5): p. 551-565.

23. Alshaikh, E.M., et al., The potential influence of hyperthyroidism on circulating adipokines chemerin, visfatin, and omentin. Int J Health Sci (Qassim), 2019. 13(2): p. 44-47.

24. Brannmark, C., et al., Adiponectin is secreted via caveolin 1-dependent mechanisms in white adipocytes. J Endocrinol, 2020. 247(1): p. 25-38.

\section{Figures}




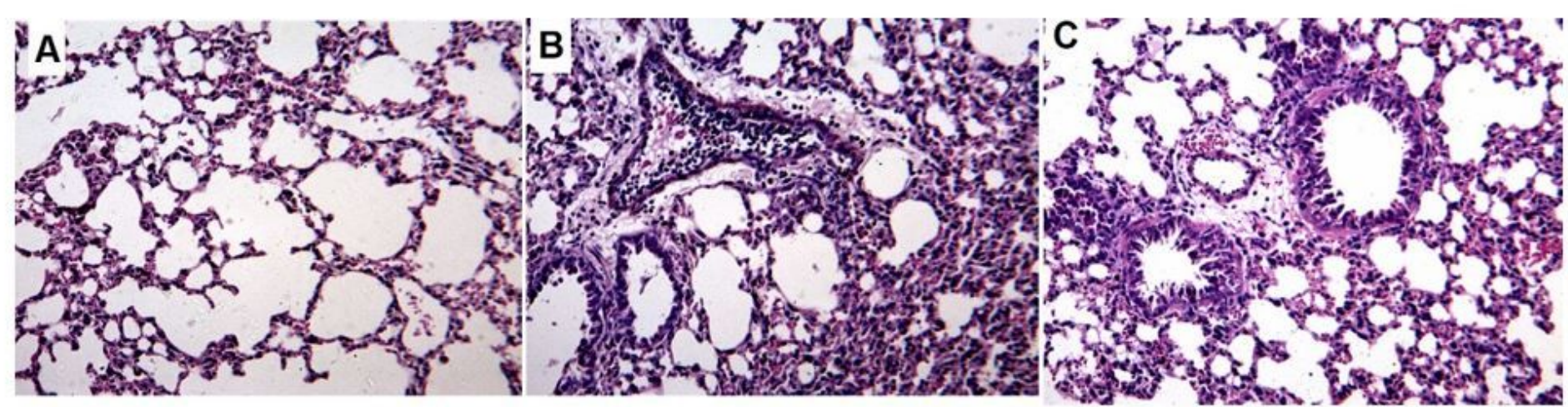

D

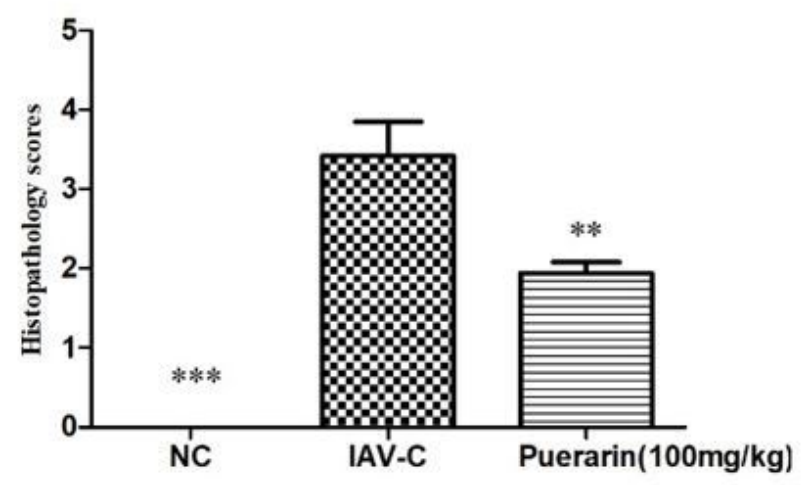

E

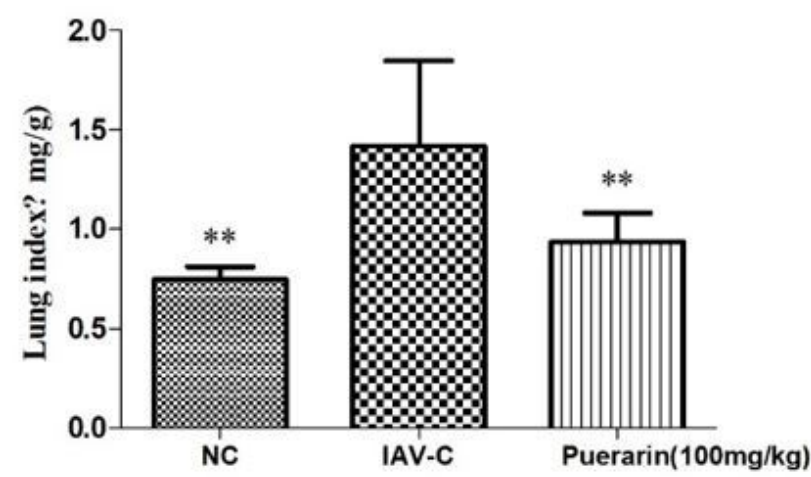

Figure 1

Pathological changes of lungs in IAV-infected mice at day 6 after-infection $(n=6-10)$. a Pathological changes of mice lung tissues in $\mathrm{NC}$ group $(\mathrm{HE}, \times 100)$; b: Pathological changes of mice lung tissues in IAV-C group (HE, * 100); c: Pathological changes of mice lung tissues in puerarin group (HE, * 100); d pathological scores(0 represented no pneumonia; 1 mild interstitial pneumonia, $<25 \%$ of the lung; 2 moderate interstitial pneumonia, $25-50 \%$ of the lung; 3 severe interstitial pneumonia, $50-75 \%$ of the lung, and 4 very severe interstitial pneumonia, $>75 \%$ of the lung). e Lung index. Data were presented as mean \pm SD. Asterisks denote the significance levels: ${ }^{* \star} \mathrm{p}<0.01$; ${ }^{* \star} \mathrm{p}<0.001$, compared with IAV-C group.

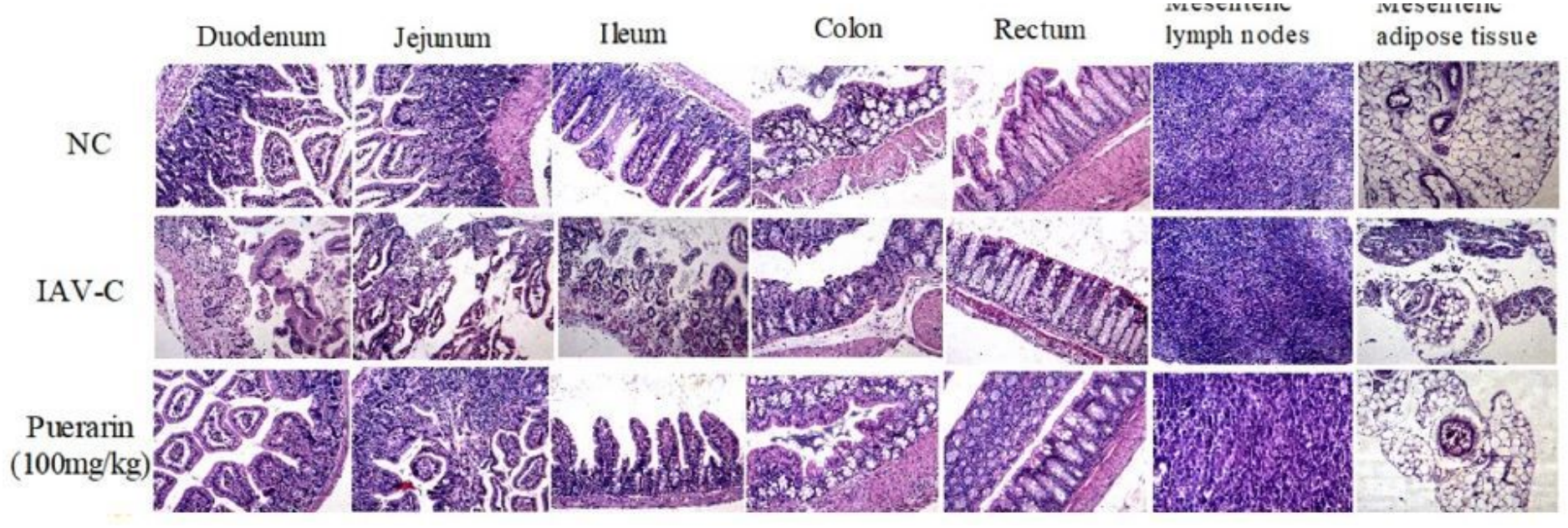

Figure 2 
Pathological changes of intestine in IAV-infected mice virus at day 6 after-infection $(n=6-10)(H E, * 100)$.
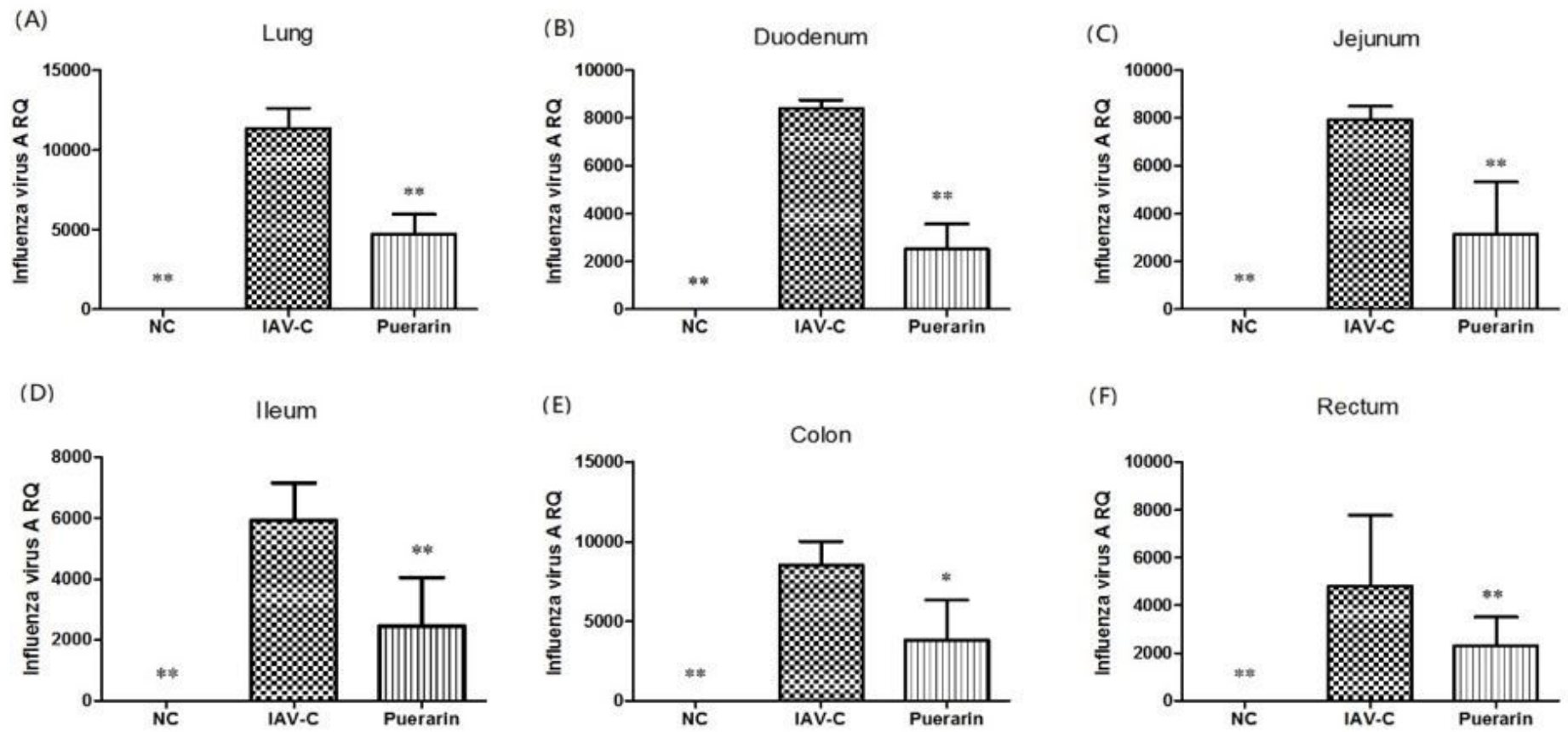

(F)

Rectum
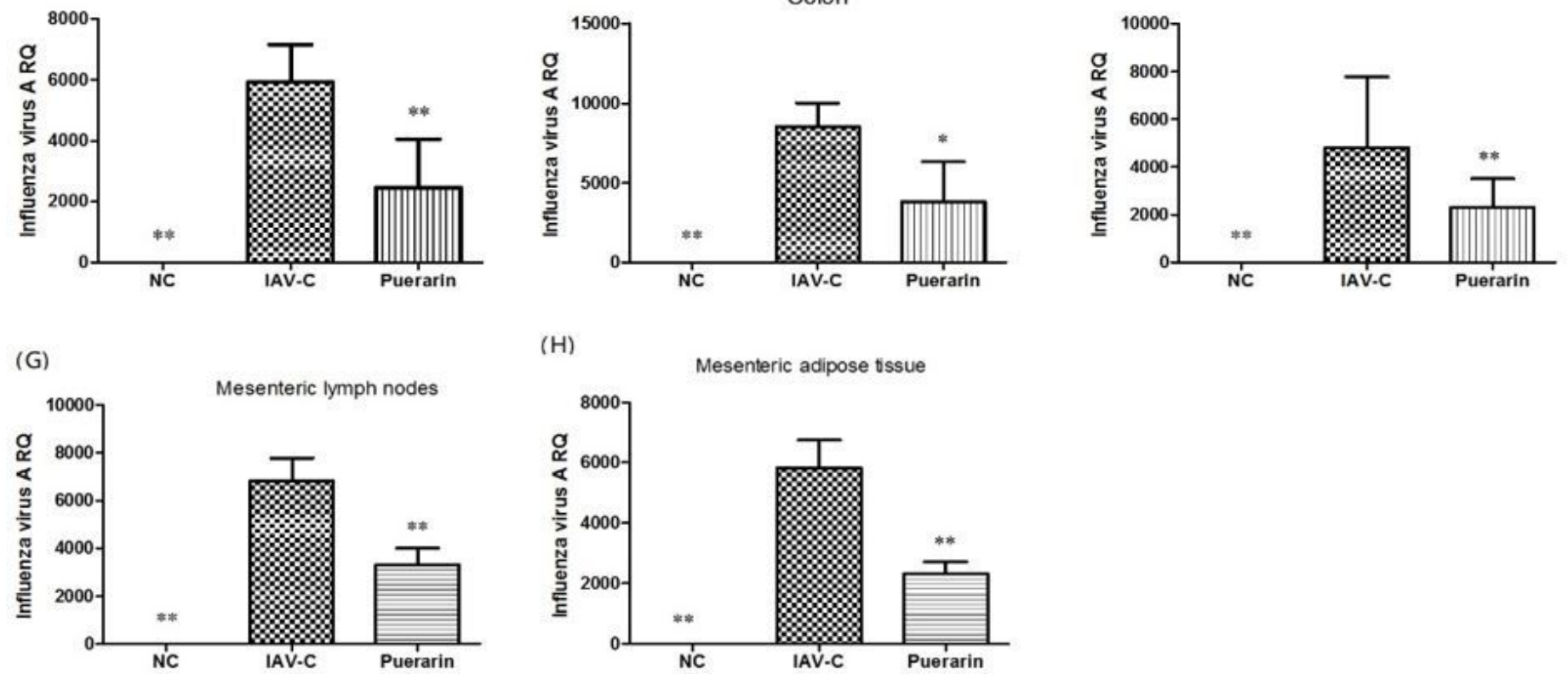

\section{Figure 3}

Changes of viral RNA level after intranasal infection of influenza virus in mice ( $n=6-10)$. a Relative quantitation of influenza A virus in lung; b: Relative quantitation of influenza A virus in duodenum; c: Relative quantitation of influenza A virus in jejunum; $d$ Relative quantitation of influenza A virus in ileum; e Relative quantitation of influenza A virus in colon; $f$ Relative quantitation of influenza A virus in rectum; $g$ Relative quantitation of influenza A virus in mesenteric lymph nodes; $h$ Relative quantitation of influenza A virus in mesenteric adipose tissue. Data were presented as mean \pm SD. Asterisks denote the significance levels: ${ }^{\star} \mathrm{p}<0.05 ;{ }^{*} \mathrm{p}<0.01$, compared with IAV-C group. 
(A)

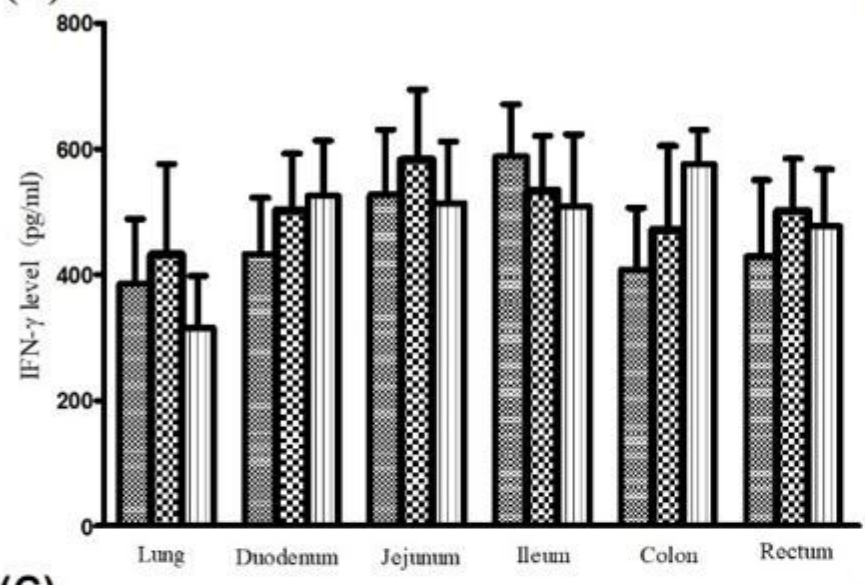

(C)

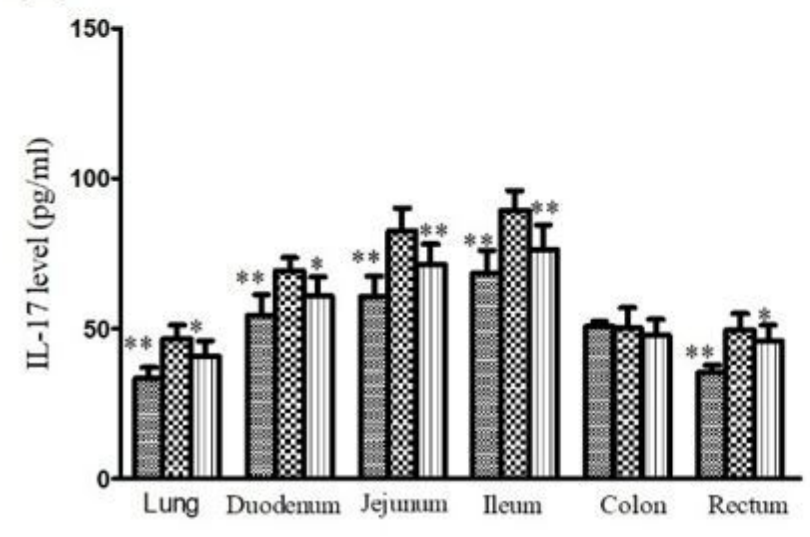

(B)

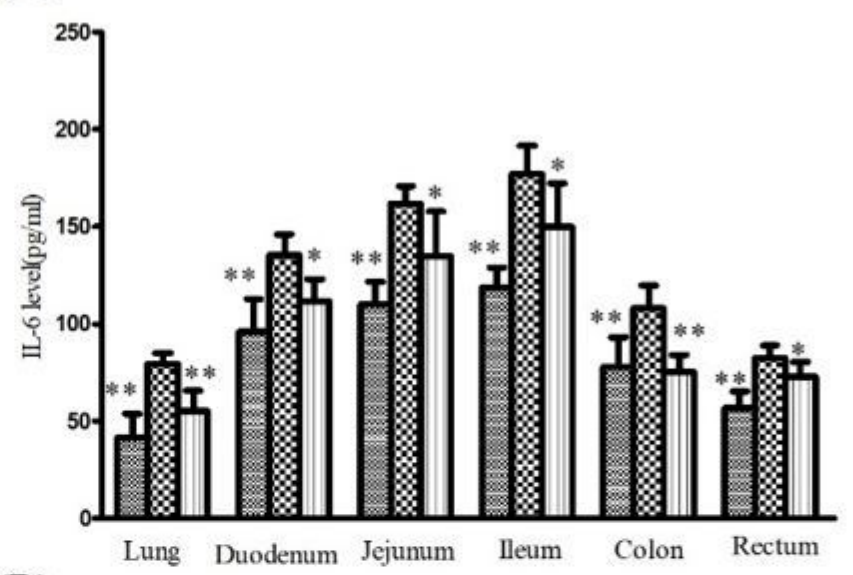

(D)

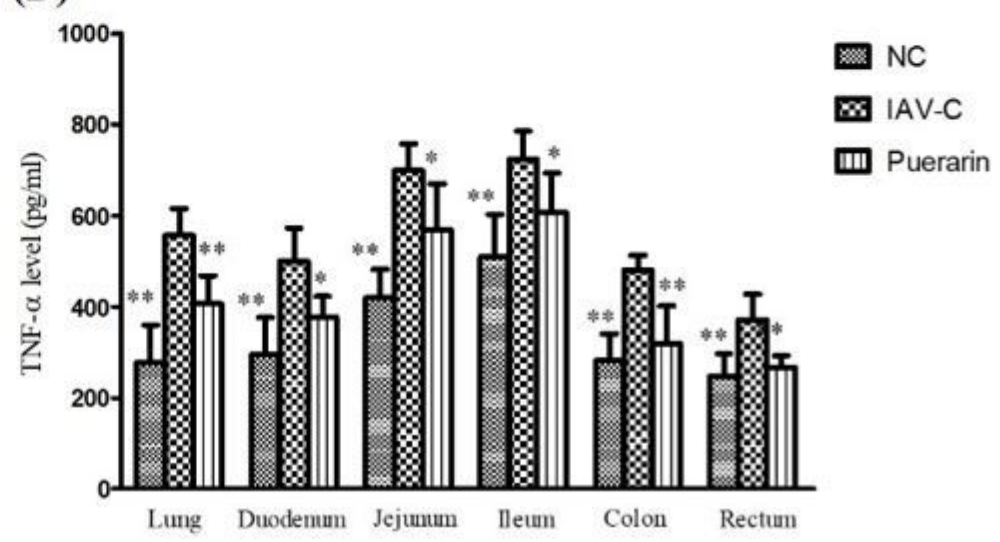

Figure 4

Changes of inflammatory cytokines in lung and intestine of IAV-infected mice $(n=10)$. Proinflammatory cytokine levels were analyzed on day 6 after infection by ELISA. a The level of IFN-gamma; $b$ The level of IL-6; c The level of IL-17; $d$ The level of TNF-alpha. Data were presented as mean \pm SD. Asterisks denote the significance levels: ${ }^{\star} p<0.05 ;{ }^{\star \star} p<0.01$, compared with IAV-C group. 


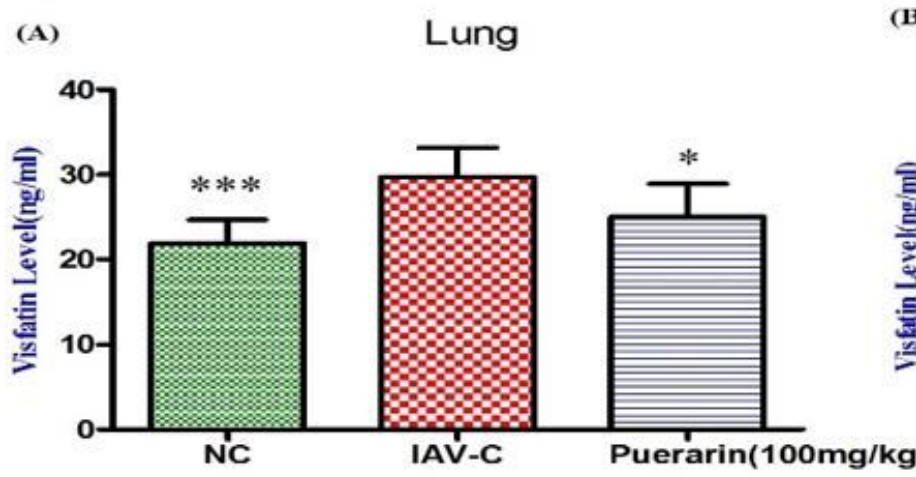

(B)

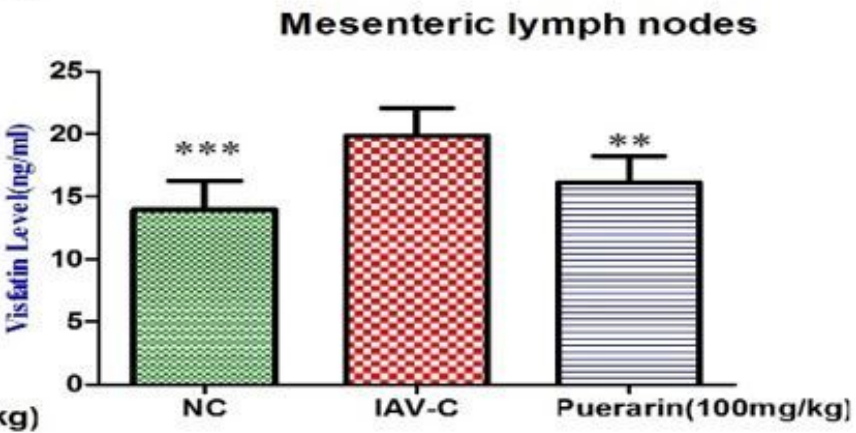

(C)

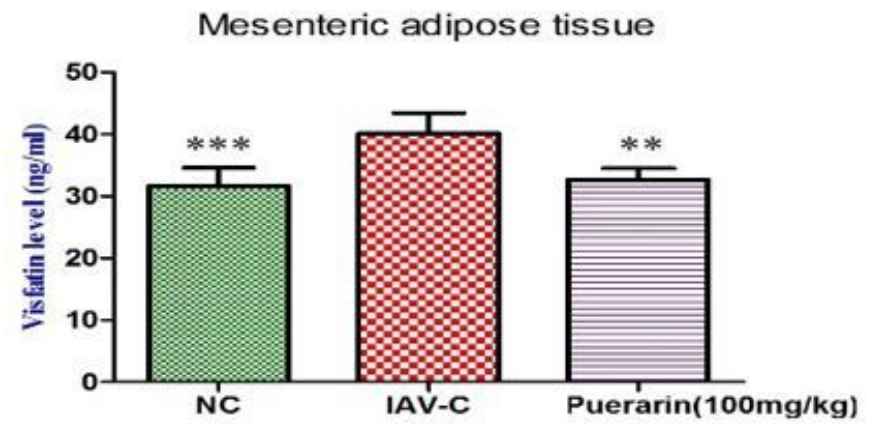

Figure 5

Changes of visfatin in lung and intestine of IAV-infected mice $(n=10)$. Visfatin levels were analyzed on day 6 after infection by ELISA. a The level of visfatin in lungs; $b$ The level of visfatin in mesenteric lymph nodes; $c$ The level of visfatin in mesenteric adipose tissue. Data were presented as mean \pm SD. Asterisks denote the significance levels: ${ }^{\star} p<0.05 ;{ }^{*} p<0.01 ;{ }^{* \star *} p<0.001$, compared with IAV-C group. 
(A)

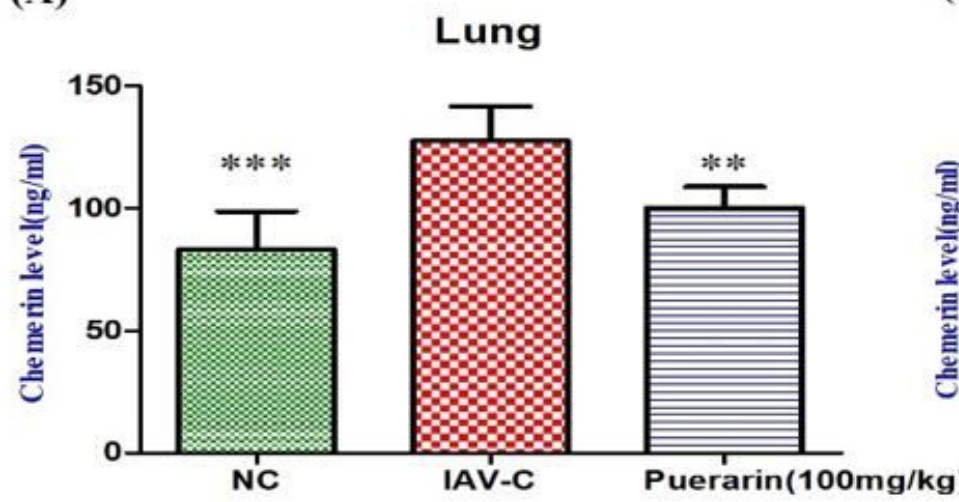

(B)

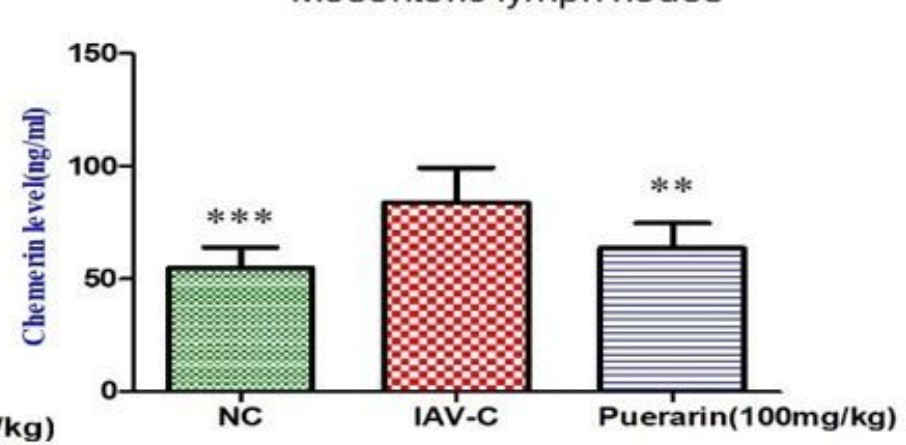

(C)

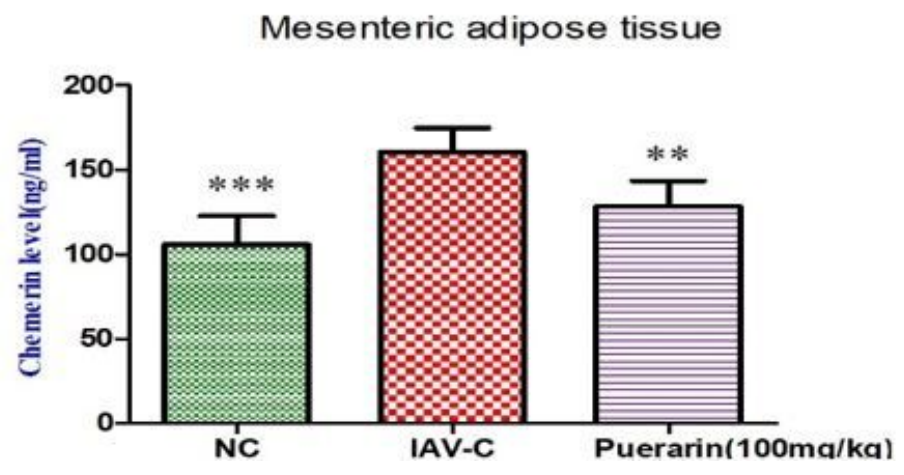

Figure 6

Changes of chemerin in lung and intestine of IAV-infected mice $(n=10)$. Chemerin levels were analyzed on day 6 after infection by ELISA. a The level of chemerin in lungs; $b$ The level of chemerin in mesenteric lymph nodes; c The level of chemerin in mesenteric adipose tissue. Data were presented as mean \pm SD. Asterisks denote the significance levels: ${ }^{*} \mathrm{p}<0.01$; ${ }^{* \star} \mathrm{p}<0.001$, compared with IAV-C group. 
(A)

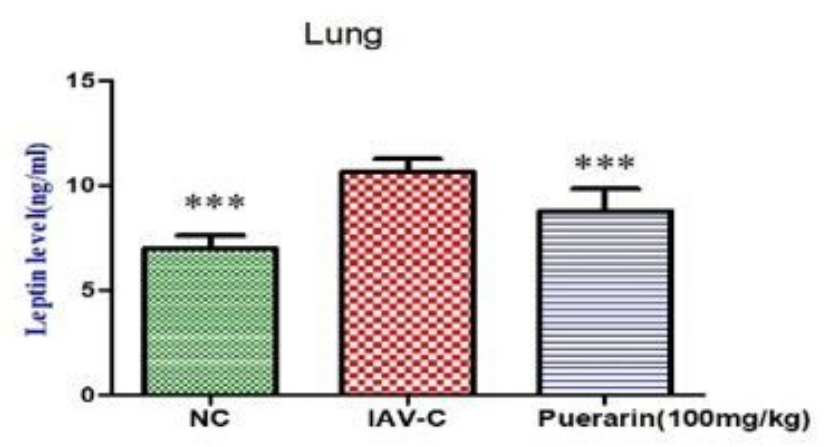

(C)

Mesenteric adipose tissue

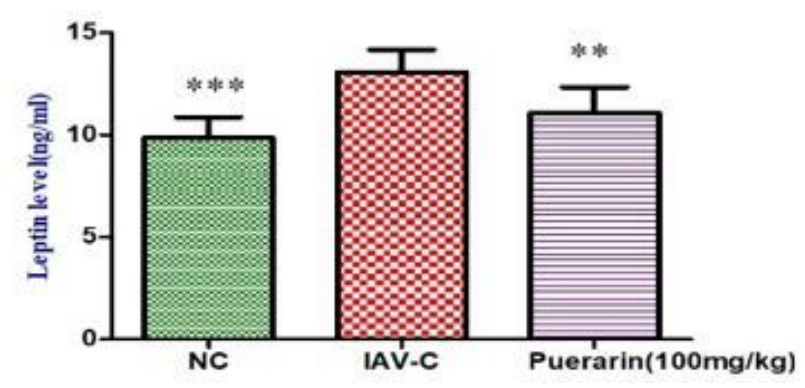

(B)

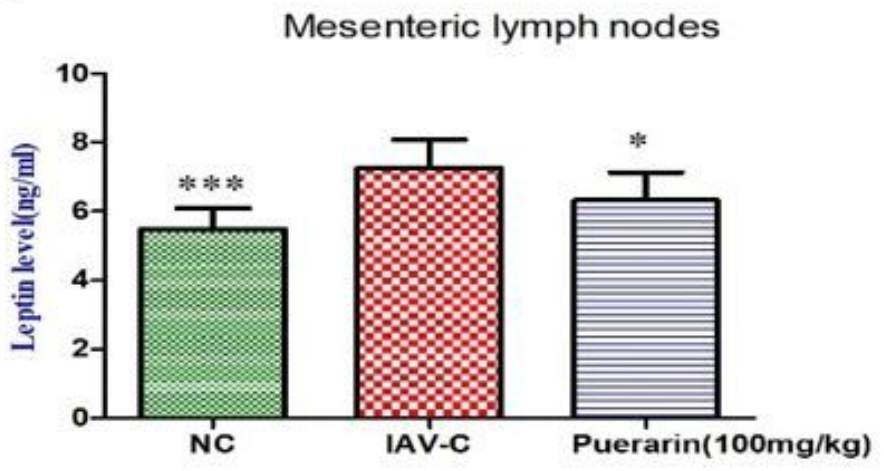

\section{Figure 7}

Changes of leptin in lung and intestine of IAV-infected mice $(n=10)$. Leptin levels were analyzed on day 6 after infection by ELISA. a The level of leptin in lungs; $b$ The level of leptin in mesenteric lymph nodes; $c$ The level of leptin in mesenteric adipose tissue. Data were presented as mean \pm SD. Asterisks denote the significance levels: ${ }^{*} p<0.05 ;{ }^{* \star} p<0.01 ;{ }^{* \star} p<0.001$, compared with IAV-C group. 
(A)

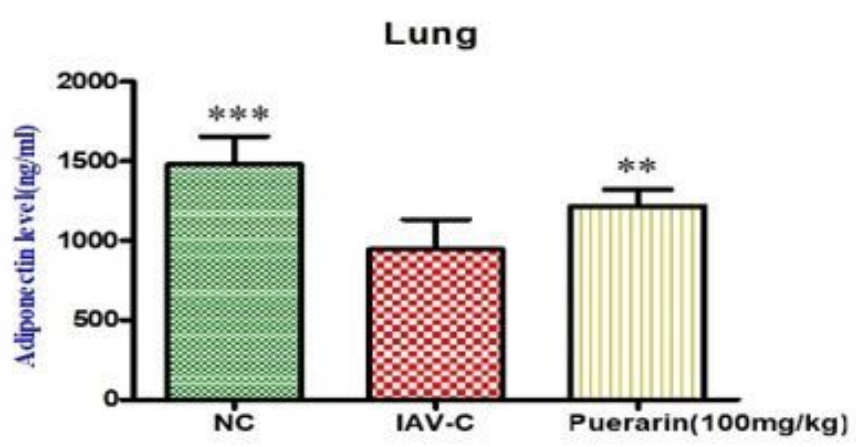

(B)

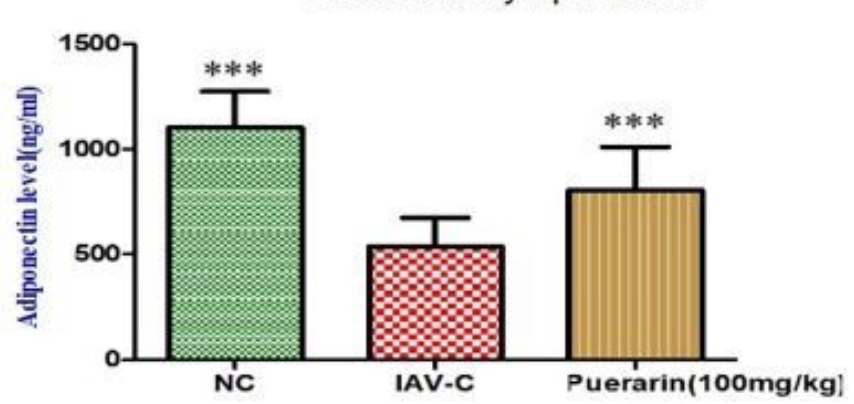

(C)

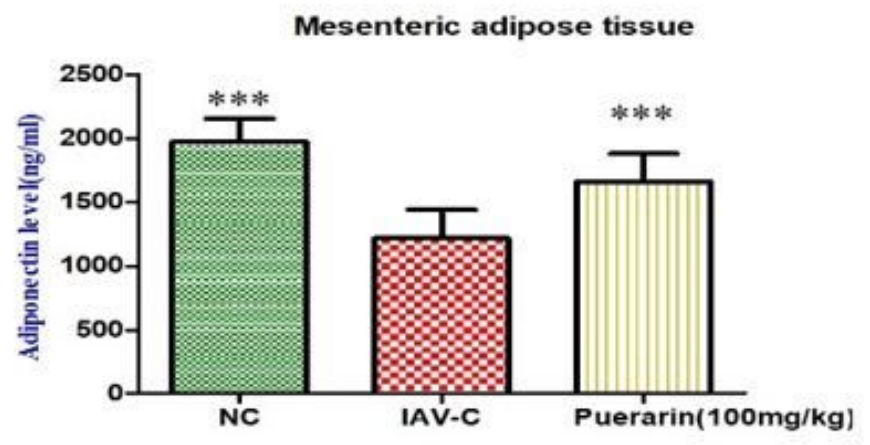

Figure 8

Changes of adiponectin in lung and intestine of IAV-infected mice $(n=10)$. Adiponectin levels were analyzed on day 6 after infection by ELISA. a The level of adiponectin in lungs; $b$ The level of adiponectin in mesenteric lymph nodes; $c$ The level of adiponectin in mesenteric adipose tissue. Data were presented as mean \pm SD. Asterisks denote the significance levels: ${ }^{*} \mathrm{p}<0.01$; ${ }^{\star \star \star} \mathrm{p}<0.001$, compared with IAV-C group.

(A)

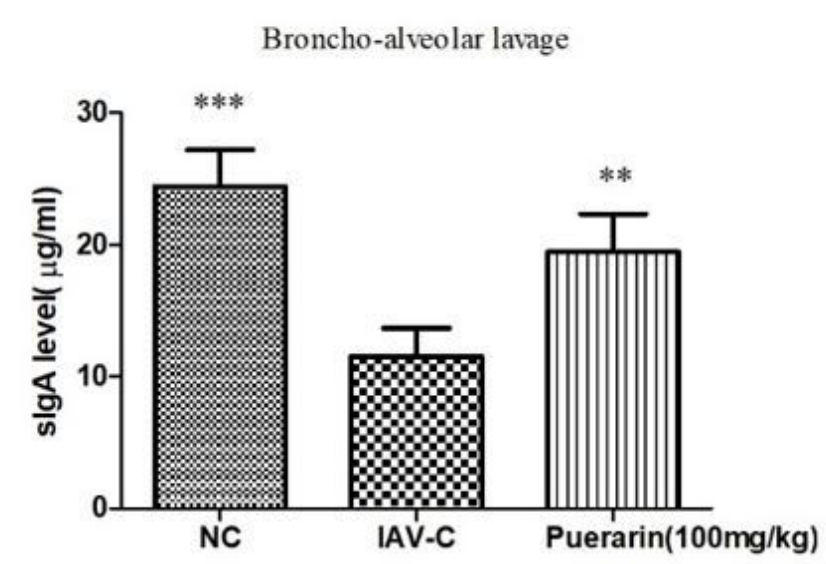

(B)

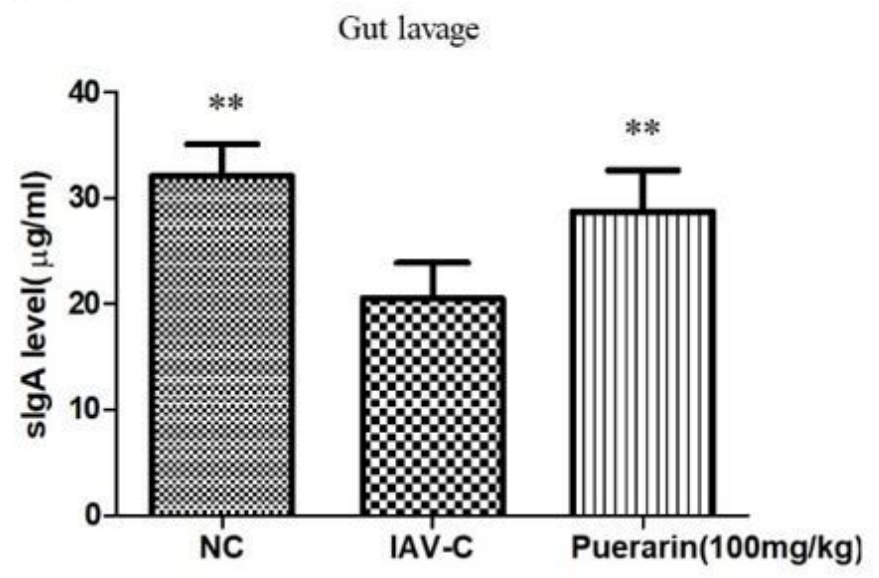

Figure 9 
IgA levels in broncho-alveolar lavage and intestinal lavage $(\mathrm{n}=10)$. The samples were collected on before infection and the 5th day post-infection. ELISA was used to determine mucosal IgA antibody levels. Data were presented as mean \pm SD. Asterisks denote the significance levels: ${ }^{\star \star} p<0.01$; ${ }^{* \star} p<0.001$, compared with IAV-C group.

(A)

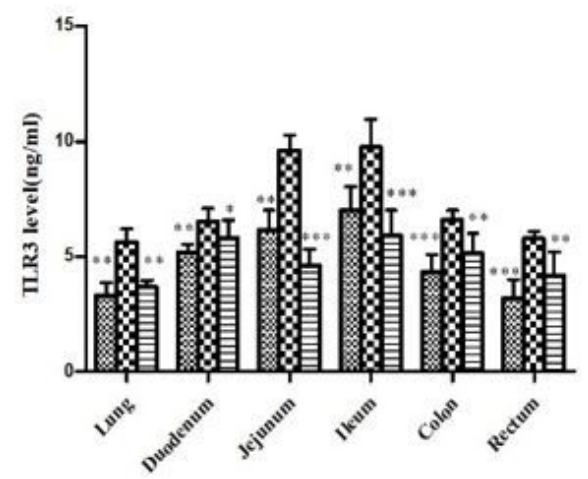

(D)

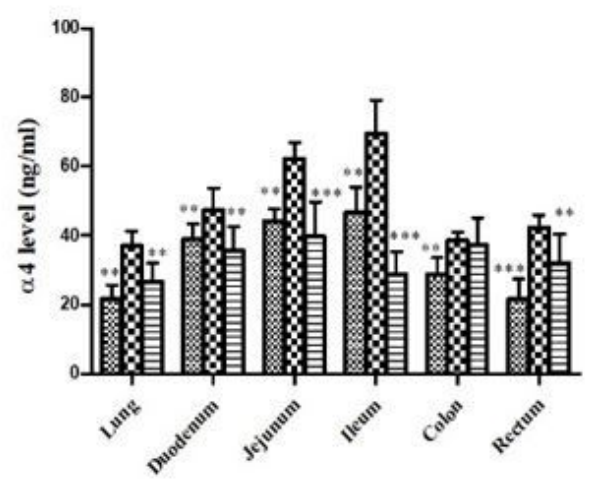

(B)

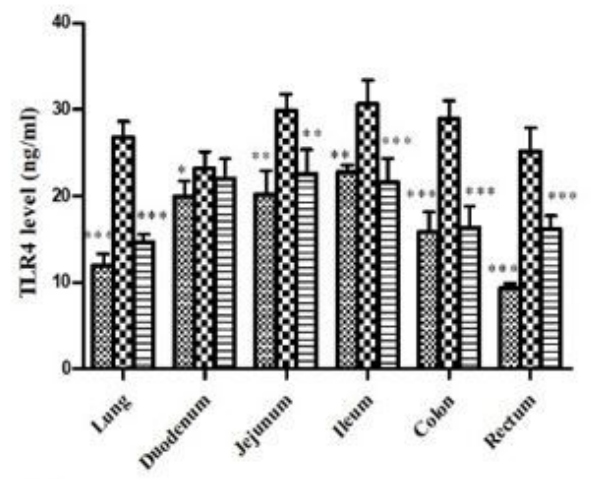

(E)

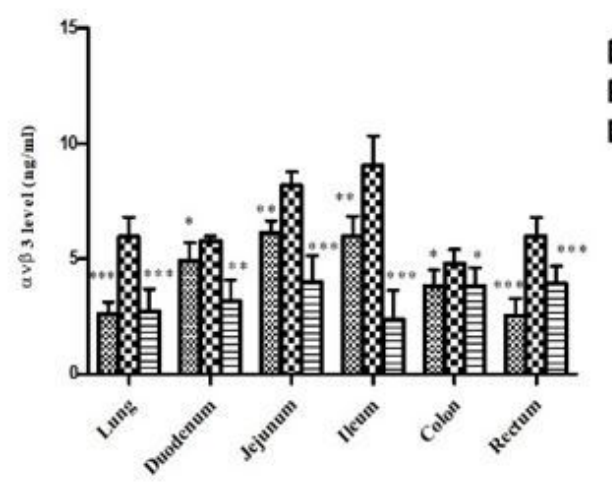

(C)

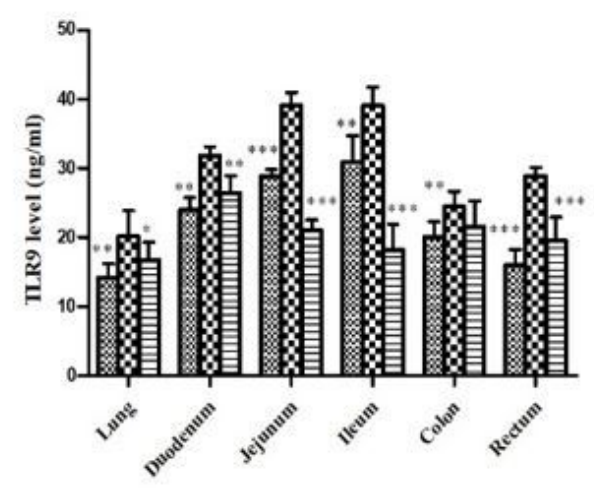

Figure 10

Changes of mucosal immune function of lung and intestine in IAV-infected mice $(n=10)$. a The level of TLR3; $b$ The level of TLR4; $c$ The level of TLR9; $d$ The level of a4; e The level of av $\beta 3$. Data were presented as mean \pm SD. Asterisks denote the significance levels: ${ }^{\star} p<0.05 ;{ }^{*} p<0.01 ; \star \star \star p<0.001$, compared with IAV-C group. 